\title{
Editorial
}

Shyam Sunder ${ }^{1}$

\section{Why Reduce Economics to Psychology}

\author{
${ }^{1}$ Yale University, New Haven, CT 06520, USA, E-mail: shyam.sunder@yale.edu
}

\begin{abstract}
:
Economics and psychology are adjacent disciplines concerned primarily with questions at aggregate and individual levels, respectively. Their interaction is useful, but attempts to integrate them into one discipline have little scientific value.
\end{abstract}

Keywords: emergence, aggregation, levels of analysis, economics and psychology

DOI: 10.1515/ael-2017-0065

Human achievements comprise science, art, the humanities, social and behavioral sciences, and professional disciplines such as medicine and engineering. Our civilization has achieved much, and led us to want more. These advances were possible through intensive interaction and interchange among the disciplines into which we have organized what we know. Although we have gained much from looking across the disciplinary boundaries, neither the delineation of precise boundaries among disciplines, nor the attempts to integrate multiple disciplines into a single unified body of knowledge have been successful. The arguments for and against unification over the recent centuries appear to have settled in a draw.

The yearning for the goal of explaining aggregate system-level phenomena in terms of the properties of individual parts persists and continues to present itself in various guises. Economics is no exception. Herbert A. Simon documented the boundedly-rational nature of human behavior, and presented evidence for satisficing as the alternative to the optimizing assumption of neoclassical economic theory. However, Simon recognized the perils of succumbing to the temptations of ignoring emergence (aggregate level phenomena that arise from micro-level interactions among many parts but whose properties cannot be derived from the properties of those parts), and refused:

This skyhook-skyscraper construction of science from the roof down to the yet unconstructed foundations was possible because the behavior of the system at each level depended on only a very approximate, simplified, abstracted characterization of the system at the level next beneath. This is lucky, else the safety of bridges and airplanes might depend on the correctness of the "Eightfold Way" of looking at elementary particles. (Simon 1996, p. 16)

Like physics and chemistry, psychology and economics also overlap, but only partially. One operates at a microlevel (physics) relative to the macro-level of the other (chemistry). Chemical phenomena are often not derivable from the physics at the micro-level. Complex interactions of components give rise to macro properties absent in the components. Physics and chemistry are closely related, and yet their models are based on different sets of assumptions. Complexity theory analyzes this phenomenon (Sayama 2015).

Economics also builds simple models (often based on optimization assumption) to try to understand, describe, and predict complex phenomena - such as a market for coffee beans - in which millions of farmers, consumers, and intermediaries participate. These models explain a significant fraction of variation, but are unable to explain all the variation observed in the relevant market phenomena. In contrast, modeling millions of individuals who participate in the market for coffee in varied roles, and interactions among them is far more difficult to construct and validate, and has little predictive power.

The less than perfect success rate of economic models has generated a strange new demand for economics to show that all its assumptions are descriptively valid, in Simon's words, "at the level next beneath." He knew that the attempts to reduce economics to psychology will fail. The deep intellectual roots of psychology are firmly grounded in individual behavior, while the primary concerns of anthropology, economics, political science, and sociology are located at the next level of aggregation.

Just as physics contributes to chemistry, so does psychology to the more aggregate-level social sciences. But the latter cannot be derived from psychology. Indeed, research in recent decades shows that aggregate level social structures, even when populated by minimally intelligent participants, often exhibit important properties absent in the individual agents (Gode \& Sunder, 1993). The properties of water molecules have little in common

Shyam Sunder is the corresponding author.

(c) 2018 Walter de Gruyter $\mathrm{CmbH}$, Berlin/Boston.

This content is free. 
with those of hydrogen and oxygen; and the hydraulic properties of water in a cup, river or ocean bear little resemblance to the perpetual random Brownian motion of its molecules. Cognitive imperfections of individuals do not preclude rational social order. Should we prefer to abandon our "irrational" autonomy and trust the philosopher-kings of our age - Putin, $\mathrm{Xi}$, or Trump - to "nudge" us to do behave right? (Thaler \& Sunstein, 2008).

\section{References}

Gode, Dhananjay K., \& Sunder, S. (1993). Allocative efficiency of markets with zero intelligence traders: Market as a partial substitute for individual rationality. The Journal of Political Economy, 101(1), 119-137.

Sayama, Hiroki. (2015). Introduction to the modeling and analysis of complex systems. Retrieved from https://textbooks.opensuny.org/introduction-to-the-modeling-and-analysis-of-complex-systems/.

Simon, Herbert A. (1996). The sciences of the artificial (3rd ed.). MIT Press. ISBN-13: 978-0262691914.

Thaler, Richard H, \& Sunstein, Cass R. (2008). Nudge: Improving decisions about health, wealth, and happiness. Yale University Press. ISBN 9780-14-311526-7. 